\title{
The Potential of Drug Delivery Nanosystems for Sepsis Treatment
}

\author{
Yi Shi ${ }^{1, *}$ \\ Meng-Lu Zhu ${ }^{2, *}$ \\ Qian Wu' \\ Yi Huang' \\ Xiao-Ling $\mathrm{Xu}^{3}$ \\ Wei Chen'
}

'ICU, Longhua Hospital Affiliated to Shanghai University of Traditional Chinese Medicine, Shanghai, 200032, People's Republic of China; ${ }^{2}$ Department of Pharmacy, Fourth Affiliated Hospital, School of Medicine, Zhejiang University, Yiwu, 310006, People's Republic of China; ${ }^{3}$ Shulan International Medical College, Zhejiang Shuren University, Hangzhou, 310004, People's Republic of China

*These authors contributed equally to this work
Correspondence: Wei Chen

ICU, Longhua Hospital Affiliated to Shanghai University of Traditional Chinese Medicine, 725 South WanPing Road, Shanghai, 200032, People's Republic of China

Tel +86-02I-64385700-3522

Email cwdoctor@shutcm.edu.cn

Xiao-Ling Xu

Shulan International Medical College,

Zhejiang Shuren University, 8 Shuren

Street, Hangzhou, 310004, People's

Republic of China

Email ziyao1988@zju.edu.cn

\begin{abstract}
Sepsis is a major immune response disorder caused by infection, with very high incidence and mortality rates. In the clinic, sepsis and its complications are mainly controlled and treated with antibiotics, anti-inflammatory, and antioxidant drugs. However, these treatments have some shortcomings, such as rapid metabolism and severe side effects. The emergence of drug delivery nanosystems can significantly improve tissue permeability, prolong drugs' circulation time, and reduce side effects. In this paper, we reviewed recent drug delivery nanosystems designed for sepsis treatment based on their mechanisms (anti-bacterial, anti-inflammatory, and antioxidant). Although great progress has been made recently, clinical practice transformation is still very difficult. Therefore, we also discussed key obstacles, including tissue distribution, overcoming bacterial resistance, and single treatment modes. Finally, a rigorous optimization of drug delivery nanosystems is expected to present great potential for sepsis therapy.
\end{abstract}

Keywords: sepsis, anti-bacterial, anti-inflammatory, anti-oxidative, drug delivery nanosystems, multidrug resistance

\section{Introduction}

Sepsis, a life-threatening organ dysfunction caused by the host immune response to infection, is one of the leading causes of death worldwide. According to a recently published study, ${ }^{1} 48.9$ million sepsis cases and 11 million sepsis-related deaths were recorded in the world in 2017 , accounting for about $20 \%$ of global deaths. ${ }^{2}$ Among them, septic shock and multiple organ dysfunction mortality can be as high as $50 \%$. Although critically ill sepsis patients' management has improved in the past decade, sepsis-related mortality is still high. ${ }^{3,4}$ The global age-standardized sepsis incidence rate dropped from 1074.7 cases per 100,000 in 1990 to 67.5 cases per 100,000 in 2017, showing a decrease of 37.0\%. The percentage of global deaths related to age-standardized sepsis dropped from $29.1 \%$ in 1990 to $20.1 \%$ in 2017 , indicating a decrease of $31.0 \%{ }^{5}$ Moreover, the prognosis of sepsis is severe. A total of $30 \%$ of survivors can develop long-term dysfunction and cognitive impairment, ${ }^{6}$ leading to a tremendous burden to society and individuals.

Sepsis management guidelines focus on three main components: ${ }^{7}(1)$ hemodynamic stability, (2) infection control, and (3) sepsis response regulation. Other interventions include organ support non-specific measures, such as oxygen therapy, mechanical ventilation, hemodynamic support, corticosteroids, and renal replacement therapy. ${ }^{8}$ Often, sepsis management is based on multimodal treatment severity. For example, mild single organ dysfunction can be controlled by appropriate support, while multiple organ dysfunction requires invasive treatments. 
Antibiotic treatments are the standard in clinical guidelines for sepsis. However, $70-80 \%$ of sepsis deaths are related to persistent infection, indicating widespread antibiotic resistance and a lack of effective antibiotics. For example, it is estimated that 214,000 neonatal septicemia deaths are caused by drug-resistant pathogens worldwide. ${ }^{9,10}$ In neonatal drug resistance, Escherichia coli showed resistance to $\beta$-lactam antibiotics and aminoglycosides; group $B$ streptococci were resistant to penicillin; Listeria monocytogenes were resistant to cephalosporins and vancomycin; Staphylococcus aureus and coagulase-negative staphylococci were not sensitive to vancomycin and methicillin. ${ }^{11,12}$ This drug resistance can harm sepsis prognosis and almost double mortality.

Additionally, although antibiotics' timely administration is very active, the host sepsis response can trigger the systemic release of various cytokines, reactive oxygen species, and other biomolecules. This can be overwhelming and easily lead to multiple organ failures and death. Recent clinical data showed that despite more than $60 \%$ of sepsis patients surviving the initial inflammatory storm, they rapidly developed a longer immunosuppressive state, characterized by paralysis and immune cells' death, resulting in the inability to remove invasive pathogens increasing hospital-acquired infection susceptibility, and high mortality. Therefore, new adjuvant therapies such as anti-inflammatory agents, immunomodulators, and antioxidants are being explored. However, many antiinflammatory agents and antioxidants are limited by a short half-life, lack of tissue or cell-specific targeting ability, and poor water solubility and bioavailability. Additionally, due to cellular enzymes activities, some peptides showed significant anti-inflammatory activity in vitro but could not reproduce this effect in vivo. Additionally, complex pathophysiology involving multi-channel cytokine storms requires a multi-pronged approach since single-drug approach may not be effective.

The emergence of nanotechnology has opened up a new pathway to overcome the serious adverse effects and resistance of drugs. Nanotechnology is a system that takes synthetic or natural materials as carriers and introduces drugs through various methods (grafting, adsorption, and physical encapsulation), which is called a drug-delivery nanosystem. Besides this, nanotechnology can also include nano-drug crystals that directly process raw drugs. This review only focuses on drug-delivery nanosystems. Most nanocarriers do not have any function (some special nanocarriers may show certain internal activities, such as photothermal performance, antioxidant performance, etc.), while drugs have nano-size and surface characteristics because they enter a new system, which significantly changes their own distribution and metabolic pathway to play a drug effect different from raw drugs. Different nanocarriers have been developed, including liposomes, ${ }^{13,14}$ nanoparticles, ${ }^{15}$ solid lipid nanoparticles, ${ }^{16}$ polymer micelles, ${ }^{17,18}$ and extracellular vesicles. ${ }^{19-21}$ The employment of nanotechnology has great potential for accurate sepsis treatment, attributed to their proper physical and chemical properties (such as size, charge, surface chemistry, shape, etc.) and their possible surface functionalization. For example, a particle size ranging from $0.5 \mu \mathrm{m}$ to $5 \mu \mathrm{m}$ exerts an excellent pulmonary-targeting ability since the particles could be trapped by pulmonary capillaries. ${ }^{22}$ Aiming at targeting renal tubule, drug-delivery nanosystems with a particle size smaller than endothelial fenestrations (70$90 \mathrm{~nm}$ ) could be designed since nanocarriers need to pass anatomical barriers of endothelial fenestrations ( $70-90 \mathrm{~nm}$ ) and be trapped in the gap between endothelial fenestrations and the glomerular basement membrane $(2-8 \mathrm{~nm})$. Furthermore, a positively charged drug-delivery nanosystem could be transported more easily than negatively charged drug carriers owing to the presence of negatively charged heparin sulfate and sulfated glycosaminoglycan on the fenestrae. ${ }^{23,24}$ Additionally, drug-delivery nanosystems can also be engineered with peptides, ${ }^{25}$ antibodies, ${ }^{26}$ and oligonucleotides, ${ }^{27}$ allowing site-specific delivery of drugs. These contribute to modulating the pharmacokinetics of the encapsulated drug, improving the efficacy, stability, and bioavailability. $^{28}$ All of these options together have prompted drug-delivery nanosystems for the precision treatment of sepsis.

Therefore, in this review, we introduced drug delivery nanosystems designed for sepsis treatment based on different mechanisms (eg, antibacterial, antioxidative, and anti-inflammatory). Meanwhile, we concluded and discussed the future outlooks of these systems.

\section{Drug Delivery Nanosystems for Sepsis Treatments} Antibacterial Drug Delivery Nanosystems

Sepsis and its complications are mainly controlled and treated with antibiotics and other drugs. However, smallmolecule drugs have disadvantages in the organism, such as a too-fast clearance rate, low utilization, high toxicity and side effects, which may lead to inappropriate treatment 
effects. Recently, with material technology and nanotechnology's continuous development, studies involving antibacterial and anti-inflammatory drug delivery nanosystems, based on functional materials, have been published. ${ }^{28-30}$ However, how to truly target the infection site and effectively treat and control sepsis has not been solved. Based on sepsis's pathological characteristics, Zhang et $\mathrm{al}^{31}$ designed and developed bioresponsive nanoparticles for drug targeted delivery, achieving effective sepsis control and treatment. First, a pH/enzyme sensitive amphiphilic polymer was synthesized by Michael's stepby-step addition method, which could self-assemble into nano micelles and effectively load antibiotics and antiinflammatory drugs. Then, through biotin-avidin specific action, targeted intercellular adhesion molecule-1 (ICAM1) antibodies were modified on drug-loaded nano micelles' surface, and new drug delivery nanosystems with targeting effect were prepared. To evaluate biocompatibility, the cell viability of the prepared nanosystem was assessed in three cell lines (NHF, HUVEC, and HEK 293T). There was no obvious change in the percentages of survival cells after nanosystem treatment, implying nonsignificant cytotoxicity. In a sepsis mouse model caused by bacteria, the drug delivery nanosystems effectively eliminated the invasive bacteria and alleviated the inflammatory reaction, thus improving the survival rate. Altogether, these results confirmed the capability of the new drug delivery nanosystems for effective sepsis treatment.

Due to a serious increase in bacterial resistance, treating sepsis caused by drug-resistant bacteria infections is even more difficult. Macrophages, one of the main participants in host immune defense, mainly encapsulate bacteria in its phagocytic lysosomes, and kill bacteria through active nitrogen oxides and lysosomal enzymes activity. However, many bacteria, such as Staphylococcus aureus and Escherichia coli, can survive in cells, resisting bactericidal mechanisms and eventually leading to infection recurrence. Additionally, although antibiotic therapies are among the most basic treatments for bacterial sepsis, $70-80 \%$ of sepsis deaths are still accompanied by persistent infection, which might be due to a lack of effective antibiotics for some drug-resistant bacteria. To solve the drug-resistance problem, Hou et $\mathrm{al}^{32}$ designed and constructed an mRNA encoding an antimicrobial peptide, an enzyme-sensitive linker peptide, and a lysosomal signal protein. They screened lipidic vitamins with optimal composition to prepare vitamin $\mathrm{C}$ nanoparticles. After the nanoparticles enter the macrophages, the mRNA is released into the cytoplasm, then translated into a triblock protein: antimicrobial peptide enzyme-sensitive peptide lysosomal signal protein. Under lysosomal signal protein guidance, the triblock protein enters the lysosome, and the sensitive enzyme peptide is cut off by lysosomal enzymes, and the antimicrobial peptide is finally released. When the macrophage contacted the bacteria, bacteria were first wrapped in the phagosome, and then the phagosome fused with the lysosome. At this time, the exogenous antimicrobial peptides and other bactericidal components in the lysosome dissolved into the phagosome, achieving a synergistic bactericidal effect, effectively reducing the number of drug-resistant bacteria in the body and improving the host's body function. Finally, the host's survival rate of sepsis induced by drug-resistant bacteria was improved. Surprisingly, the levels of bodyweight, white blood cells, and lymphocytes of the survived mice fully recovered after treatment, implying good biosafety.

The shape of nanoparticles is closely related to their biological distribution and interaction with target cells, affecting drug delivery characteristics. Anisotropic nanoparticles indicated that the shape of nanoparticles was not invariant with respect to direction. Compared with spherical nanoparticles, anisotropic ones have greater resistance to non-specific cell elimination when administered in the whole body, which would enhance the membrane-coated nanoparticles' stealth. Almost all previous bionic designs used spherical nanoparticles. To fabricate anisotropic nanoparticles, Ben Akiva et $\mathrm{al}^{33}$ first synthesized spherical poly (lactic-co-glycolic acid) (PLGA) nanoparticles by single emulsion and then stretched them above the glass transition temperature of PLGA. Stretching two-fold in one dimension generated prolate nanoparticles, and 1.5-fold in two dimensions presented oblate ellipsoidal nanoparticles. After interaction with red blood cell membranes, three types of biomimetic anisotropic nanoparticles were yielded. The results showed that although the prepared anisotropic nanoparticles curvature radius increased, it did not significantly affect coating fluidity or stability, and can be covered by naturally derived cell membranes. Compared with uncoated spherical nanoparticles, anisotropic nanoparticles coated with red blood cell membrane can better escape macrophages' clearance and reduce 50 to $60 \%$ of internalization, resulting from the synergistic effect of altering the nanoparticle shape and introduction of the membrane. Moreover, the nanoparticles with a prolate ellipsoidal shape demonstrated a superior half-life (171.6 $\mathrm{min}$ ) compared to any other nanoparticles ( $82.0 \mathrm{~min}$ for coated oblate ellipsoidal particles and $64.8 \mathrm{~min}$ for coated spherical particles). As a result, 
approximately $50 \%$ of the mice treated with coated prolate ellipsoidal nanoparticles remained healthy 1 week after alpha toxin administration, whereas only $33 \%$ of the mice in the oblate ellipsoidal nanoparticles group showed one-week survival. Overall, anisotropic nanoparticles coated with red blood membranes are expected to be a potential treatment for improving the survival rate in patients with sepsis.

Moreover, the discovery of a new antibacterial nanomaterial is another important possibility for overcoming multi-drug resistance. Since S-thanatin (Ts) is an antibacterial peptide with specific targeting ability, it was modified onto the surface of the liposome to act both as the targeting moiety and antibacterial component ${ }^{25}$ (Figure 1). Then, the antibiotic levofloxacin was loaded into liposomes with the ammonium sulfate gradient method. As displayed, Ts-anchored liposome significantly enhanced the bacterial internalization of the antibiotic, resulting in the synergistic effect of an antibiotic and antibacterial peptide. In mice receiving clinical multidrug-resistant (MDR) isolates, the bacterial was rapidly cleared by the fabricated drug delivery nanosystems, contributing to remarkably decreased lethality rate of the septic shock $(100 \%$ in the control group within $36 \mathrm{~h}$ versus $93.3 \%$ in the Ts-modified drug delivery nanosystem within $72 \mathrm{~h}$ ).

Graphene oxide (GO), a single layer with a twodimensional honeycomb lattice structure by the carbon atoms, was regarded as a promising engineered nanoplatform due to the strong antibacterial effects. Many studies have explored the bacterial killing ability of GO-based drug delivery nanosystems with different physicochemical characteristics and their mechanisms of bacterial killing. For a summary of the antibacterial applications of GObased drug delivery nanosystems, please refer to Zhang et al's review for details. ${ }^{34}$

\section{Antioxidative Drug Delivery \\ Nanosystems}

Sepsis is an abnormal systemic inflammatory response mediated by excessive reactive oxygen species (ROS) and reactive nitrogen species (RNS) production. Excessive ROS and RNS can change a series of intracellular events and the function of different enzymes and ion channels. Therefore, reducing ROS and RNS levels in cells and mitochondria can inhibit sepsis's abnormal inflammatory response. Traditional organic antioxidants have low structural stability, poor ROS and RNS scavenging activity, and poor scavenging activity persistence under physiological conditions. Yim et a ${ }^{35}$ prepared three kinds of 2D transition metal dichalcogenide nanosheets (Tungsten disulfide $\left(\mathrm{WS}_{2}\right)$, Molybdenum selenide $\left(\mathrm{MoSe}_{2}\right)$, and Tungsten diselenide ( $\left.\mathrm{WSe}_{2}\right)$ ) by liquid phasestripping using an amphiphilic copolymer (poly( $(\varepsilon-$ caprolactone)-b-poly(ethylene glycol)) (Figure 2). These exfoliated nanosheets displayed an average lateral size of

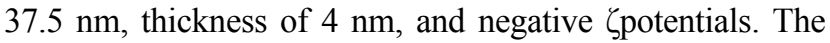
RNS and ROS scavenging experiment showed that the prepared $\mathrm{WS}_{2}, \mathrm{MoSe}_{2}$ and $\mathrm{WSe}_{2}$ nanosheets could effectively remove mitochondrial and cytosolic ROS and RNS of inflammatory cells (including hydroxyl radicals, superoxide, hydrogen peroxide $\left(\mathrm{H}_{2} \mathrm{O}_{2}\right)$, and nitric oxide). Compared with $\mathrm{MoSe} 2$ or WSe2 nanosheets, WS2 nanosheets can more effectively inhibit the excessive secretion of inflammatory cytokines except for the scavenging of ROS and RNS, contributing to significantly improved severe sepsis mice survival rate (up to 90\%). Additionally, this pharmacokinetic study showed that WS2 nanosheets could be excreted in mice after intravenous injection for 3 days.

Ceria nanoparticles exhibit similar activities to superoxide dismutase (SOD) and catalase (CAT) through reversible

\section{$-\mathrm{COOH}+\mathrm{NH2}-\mathrm{PEG} 2000-\mathrm{DSPE} \stackrel{\text { EDC }}{\longrightarrow}<-$ PEG2000-DSPE}

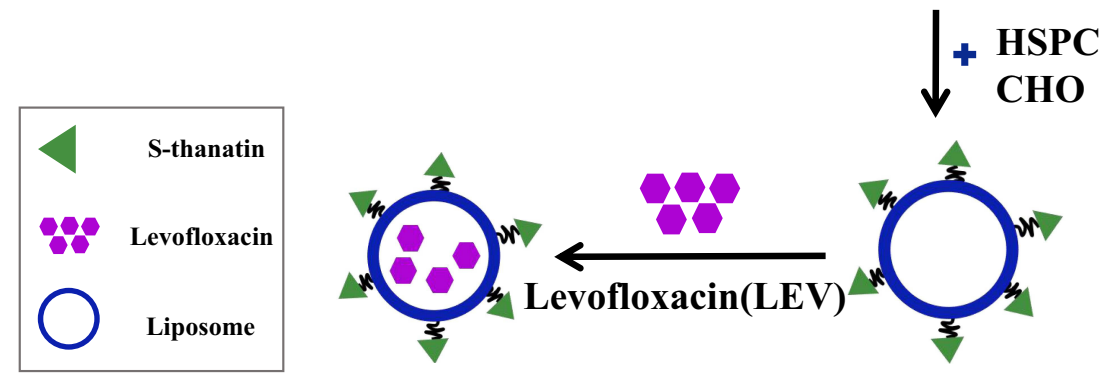

Figure I The preparation schema of S-thanatin-modifed liposomes loaded with levofloxacin. Reprinted with permission from Fan X, Fan J, Wang X, Wu P, Wu G. S-thanatin functionalized liposome potentially targeting on Klebsiella pneumoniae and its application in sepsis mouse model. Front Pharmacol. 20I5;6:249. ${ }^{25}$ 


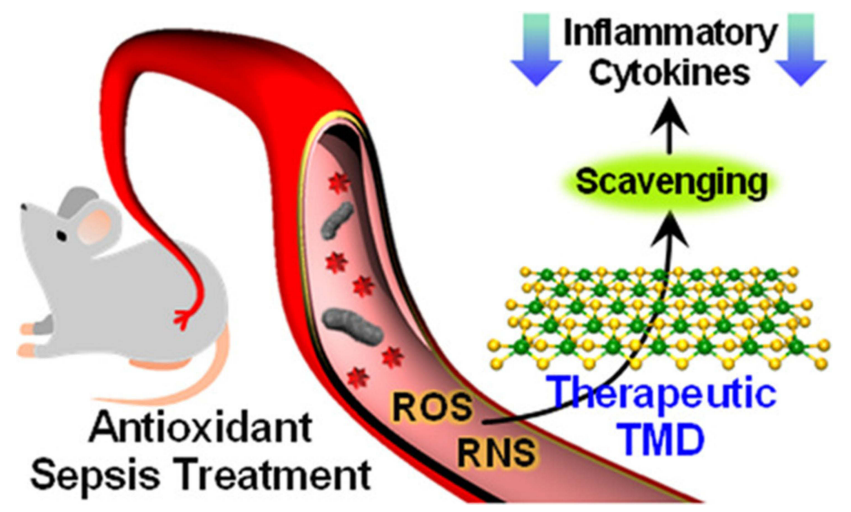

Figure 2 The treatment schema of 2D Transition Metal Dichalcogenide (TMD) Nanosheets. Reprinted with permission from Yim D, Lee DE, So Y, et al. Sustainable nanosheet antioxidants for sepsis therapy via scavenging intracellular reactive oxygen and nitrogen species. ACS Nano. 2020;14(8):10324-10336. Copyright (2020) American Chemical Society. ${ }^{35}$

conversion between $\mathrm{Ce}^{3+}$ and $\mathrm{Ce}^{4+}$ valence states and oxygen-binding. ${ }^{36,37}$ They could eliminate $\mathrm{H}_{2} \mathrm{O}_{2}$, and superoxide anion $\left(\mathrm{O}_{2-}^{-}\right)$, and hydroxyl $(\bullet \mathrm{OH})$ radicals. $^{38,39}$ Compared with most small-molecule antioxidants, cerium oxide $\left(\mathrm{CeO}_{2}\right)$ nanoparticles have a stronger ROS scavenging ability and renewable activity, promising for antioxidant applications, and have been widely studied. Chen et $\mathrm{al}^{40}$ synthesized polyphenol-stabilized $\mathrm{CeO}_{2}$ nanoparticles using Camellia sinens leaves xtract and evaluated the death rate, respiratory rate, blood pressure, and body temperature in a lipopolysaccharide (LPS)-induced sepsis model. The results demonstrated that the $\mathrm{CeO}_{2}$ treatment significantly decreased the level of tumor necrosis factor- $\alpha$ (TNF- $\alpha$ ), interleukin- $1 \beta$ (IL-1 $\beta$ ), interleukin-6 (IL-6) and high-mobility group protein 1 (HMGB1), as well as the orientation of inducible nitric oxide synthase (iNOS), an NF-kB transcriptional bustle. All these changes resulted in reduced hepatic damage and weakened systemic swelling. However, ordinary $\mathrm{CeO}_{2}$ nanoparticles can not selectively target the mitochondria, a key ROS production site in cells. Thus, it may not directly and efficiently remove excessive ROS, requiring improvement. $\mathrm{Yu}$ et $\mathrm{al}^{41}$ first synthesized triphenylphosphine-modified $\mathrm{CeO}_{2}$ nanoparticles (TCeria) to target the mitochondria, followed by the synthesis of ROS-responsive grafts. Then, atorvastatin and TCeria were co-loaded into the core of ROS-responsive grafts to construct the drug delivery nanosystems (Atv/PTPTCeria). Results showed that the prepared drug delivery nanosystems could be effectively distributed to the mice's kidneys with a sepsis-induced acute injury. Additionally, the retention time was prolonged. At 24 and $48 \mathrm{~h}$ after administration, TNF- $\alpha$ levels in renal tissue of Atv/PTP-TCeria treated mice were significantly lower, compared with the PBS group. At the same time, Atv/PTP-TCeria treatment can effectively improve renal function, alleviate renal tubular injury, and reduce renal tissue apoptosis and necrosis. Since LPS can cause liver injury in mice, the levels of serum aspartate aminotransferase and alanine aminotransferase were evaluated to confirm the compatibility. There was no significant difference between the control group and Atv/ PTP-TCeria group, suggesting that no serious adverse effect occured in the mouse liver during the treatment. This strategy provided a new idea for precisely scavenging ROS in the mitochondria of inflammatory cells.

\section{Anti-Inflammatory Drug Delivery Nanosystems}

When the human body is invaded by exogenous pathogens, lesions (tumors), or trauma, neutrophils are activated. Through self-deformation through the vascular barrier, neutrophils are enriched in the lesion.However, their survival time is short. After death, they release a large number of inflammatory factors and cause an excessive immune response. Additionally, during the human immune response, neutrophils can not distinguish between threats and non-threats. While releasing different toxic proteins to attack bacteria and other invasive pathogens, neutrophils can also kill healthy human cells, tissues, and organs, leading to various inflammatory diseases. Based on overexpressed FC- $\gamma$ on the surface of activated neutrophils, Zhang et $\mathrm{al}^{42}$ linked doxorubicin to bovine serum albumin (BSA) via $\mathrm{pH}$-sensitive bonds to prepare nanoparticles (doxorubicin-hydrazone-bovine serum albumin, DOX-hyd-BSA), which can induce apoptosis. ${ }^{43}$ The obtained nanoparticles could target and recognize activated neutrophils. When they reach the activated neutrophils, they are stimulated by low $\mathrm{pH}$, releasing anti-cancer drugs, promoting apoptosis, avoiding neutrophils' accumulation in tissues, and improving the occurrence of many inflammatory diseases, such as sepsis. Additionally, they found that mice treated with the prepared nanoparticles resumed levels of neutrophils and cytokine contents at $72 \mathrm{~h}$ comparable to the healthy mice. This indicated that DOX-hyd-BSA nanoparticles do not inhibit neutrophil production and function in bone marrow.

Lipopolysaccharide (LPS), the main structural component of the Gram-negative bacteria outer membrane, can bind to Toll-like receptor 4, triggering transcription factors

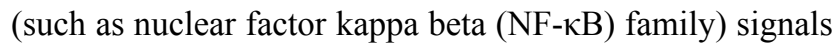
to produce inflammatory responses, the main sepsis characteristic. Curcumin (Curcuma longa) is a natural 
bisphenol compound extracted from the Curcuma longa rhizome. It has many biological and pharmacological properties such as anti-cancer and anti-inflammation. However, because of its poor water solubility, fast degradation, and low bioavailability, curcumin has a limited therapeutic effect. Therefore, Wang et $\mathrm{al}^{44}$ prepared curcumin-loaded solid lipid nanoparticles and evaluated their therapeutic potential for sepsis. The in vivo imaging results showed that in transgenic mice with LPS (3 mg/ $\mathrm{kg}$ ) intraperitoneal injection causing a corresponding inflammatory response, a maximum fluorescence intensity after 3 hours trend was detected, then gradually weakened. Intraperitoneal injection of curcumin-loaded solid lipid nanoparticles $(30 \mathrm{mg} / \mathrm{kg})$ could significantly inhibit the fluorescence expression intensity for $3 \mathrm{~h}$ and was equivalent to dexamethasone. The results of an enzyme linked immunosorbent assay showed that the curcumin-loaded solid lipid nanoparticles could significantly reduce IL-6 and TNF- $\alpha$ inflammatory factors in the serum of mice and IL-1 $\beta$. Additionally, IL-10 expression was significantly increased. The hematoxylin-eosin staining results showed that this nanosystem could effectively attenuate glomerular and tubular injury and reduce hepatic necrosis compared with the free curcumin group. Finally, Western blot results showed that it could significantly inhibit the Toll-like receptor-4 (TLR-4), TLR-2, NF- $\mathrm{BB}$ ) in lymph nodes and TNF- $\alpha$ protein expressions. These results provided new insights for sepsis treatments.

\section{Other Drug Delivery Nanosystems}

Recent studies have shown that the cell free DNA (cfDNA) content increaseds in sepsis patients' blood and can be recognized by the TLR-9 receptor of immune cells, resulting in an abnormal immune response. The TLR-9 knockout sepsis mice mortality is reduced, as the inflammation in vivo. Therefore, cfDNA neutralization or elimination may reduce sepsis organ damage by immune response regulation, inhibiting the pro-inflammatory cascade and cytokine storm. Based on this, Dawulieti et $\mathrm{al}^{45}$ synthesized three polyethyleneimine (PEI) functionalized and biodegradable mesoporous silica nanoparticles (MSN-PEI 25K, MSN-PEI 800, and MSN-NH${ }_{2}$ ) with different charge densities using PEI, and investigated their ability to scavenge cfDNA. The results showed that MSN-PEI had better therapeutic and protective effects than PEI. The therapeutic effect of MSN-PEI 25K with a high charge density on sepsis mice was better than MSN-PEI 800 with a low charge density. MSN-PEI 25K with a high charge density had a stronger nucleic acid binding ability, stronger inhibitory effect on the cfDNA induced inflammatory response, higher targeting, better retention of inflammatory sites in septic mice, and improved anti-inflammatory effects. Moreover, they found that MSNPEI 25K exhibited an increased macrophages inhibitory concentration of $61.45 \mu \mathrm{g} / \mathrm{mL}$ compared with soluble PEI $25 \mathrm{~K}$ $(22.98 \mu \mathrm{g} / \mathrm{mL})$. Additionally, MSN-PEI $25 \mathrm{~K}$ could significantly reverse the elevated serum alanine aminotransferase, aspartate aminotransferase, bilirubin, blood urea nitrogen, creatinine and creatine kinase, indicating reduced toxicity to macrophages and major organs. Therefore, it represents a more efficient and safer cfDNA scavenger.

MicroRNAs (miRNAs) are non-encoding RNAs with 2125 nucleotides, which affect gene posttranscriptional regulation by causing gene instability and preventing mRNA translation. ${ }^{46-48}$ MiR-126 is the most abundant miRNA in endothelial cells. Studies have shown that high expression of microRNA-126 (miR-126) treatment of endothelial progenitor cells can improve the survival rate of septic mice with cecal ligation and perforation. However, due to the ubiquitous rapid degradation of ribonuclease, it is challenging to transfer miRNAs to cells in vitro and in vivo. Jones Buie et $\mathrm{al}^{49}$ prepared a drug delivery system composed of deacetylated poly$\mathrm{N}$-acetyl glucosamine nanoparticles loaded with miRNA126-3p or miRNA-126-5p. The constructed nanoparticles effectively encapsulated miRNAs and prevented their migration in agarose gel, thereby protecting miRNA from Ribonuclease A (RNase A) degradation. Nearly $67 \%$ of the sepsis mice treated with the complex survived at day 7 , while only $25 \%$ of the untreated mice survived. Therefore, this drug delivery nanosystem significantly improved the survival rate and reduced inflammatory cytokines response. In addition, they also determined the impact of deacetylated polyN-acetyl glucosamine nanoparticles loaded with miRNA-126 (DEAC-pGlcNAc:miR-126) on cellular metabolic activity. No significant differences could be observed between the untreated group and the DEAC-pGlcNAc:miR-126-treated group.

All the drug delivery nanosystems developed for sepsis treatment are listed in Table 1.

\section{Conclusions}

Sepsis is a systemic inflammatory response syndrome caused by infection, with a high incidence. It has become the main cause of death for intensive care unit (ICU) patients in China and seriously threatens human health. Although the underlying sepsis pathogenesis is unclear, it is mainly related to the host's aggressive immune 


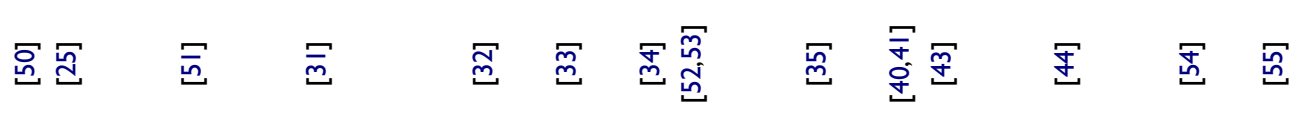

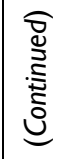

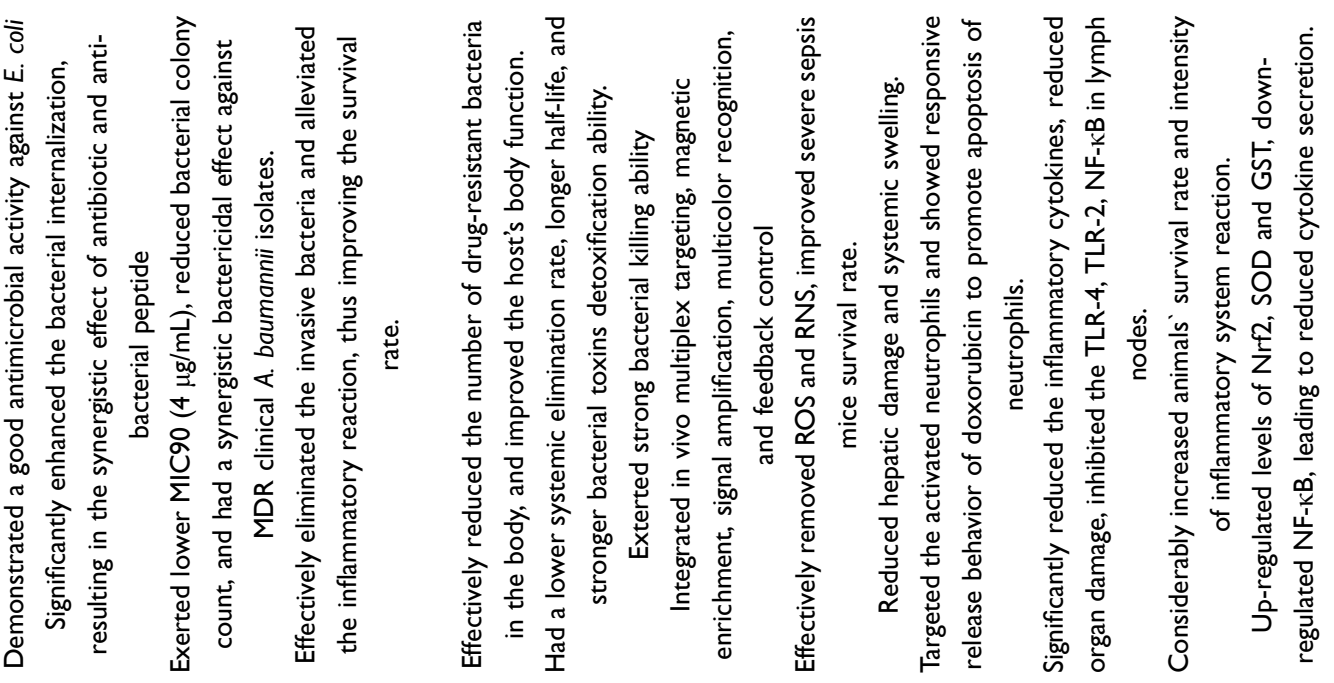

\section{ํㅗำ

(1)

政

d

$$
\text { is }
$$

.

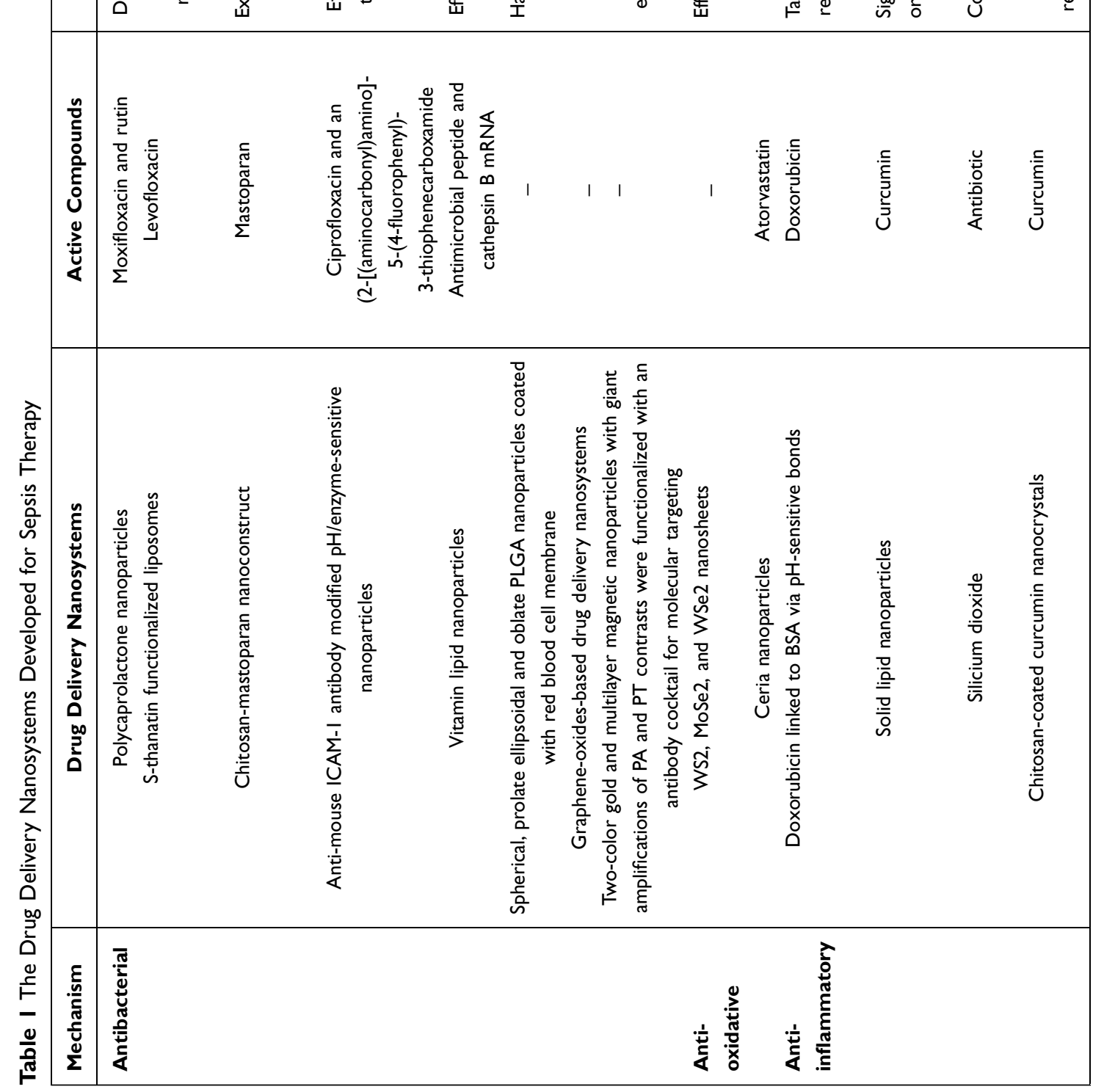




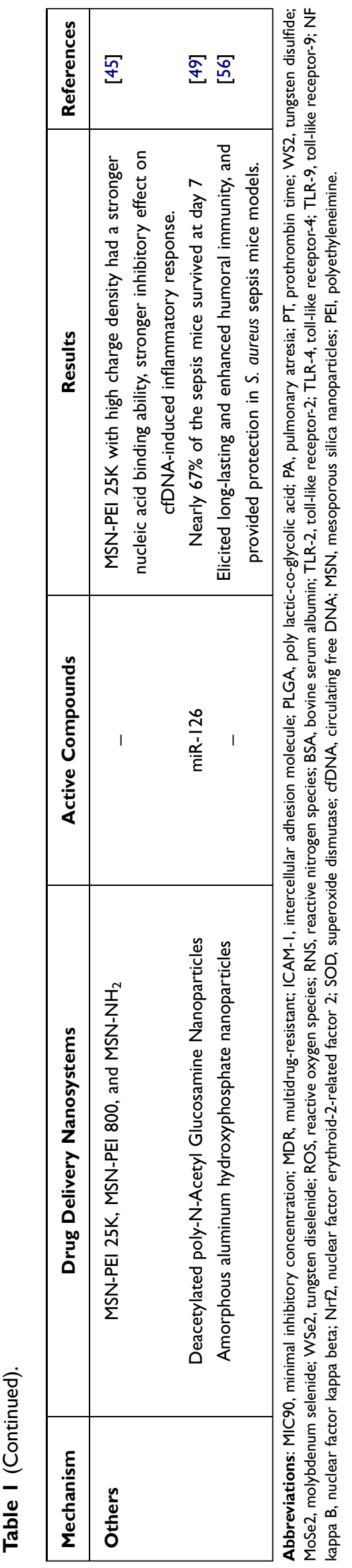

response. Therefore, sepsis and its complications are mainly controlled and treated using antibacterial, antiinflammatory, and antioxidant drugs. However, in the organism, small-molecule drugs have disadvantages, such as a too-fast clearance rate, low utilization, high toxicity and side effects, which can lead to ineffective treatments. The great advancement in nanotechnology has brought a new technological revolution for small-molecule drugs delivery. ${ }^{57-59}$ Based on action mechanisms, this paper reviewed the drug delivery nanosystems recently used for sepsis treatment. By directly targeting bacteria or inflammatory cells, these drug delivery nanosystems can significantly improve their antibacterial, anti-inflammatory, and antioxidant abilities, reducing the toxicity and side effects of long-term, high-dose administration.

There are some commercial nanoformulations for the treatment of infection, including liposomal amphotericin B (AmBisome ${ }^{\mathbb{R}}$ ), amphotericin B lipid complex $\left(\right.$ Abelcet $\left.^{\mathbb{R}}\right)$, nanosilver et al. Amphotericin B with a broad antifungal spectrum, has strong antibacterial effect on Candida, Aspergillus, and fungus. Moreover, it was reported that it had almost no drug resistance in the past. ${ }^{60}$ However, amphotericin $\mathrm{B}$ deoxycholate $(\mathrm{AmB})$, the first formulation used in the clinic, has been associated with some drawbacks, including nephrotoxicity, low solubility, and low bioavailability $(<0.9 \%) .{ }^{61}$ In contrast, nanoformulations of amphotericin $\mathrm{B}$, such as liposomal amphotericin $\mathrm{B}$, tend to prolong the circulation time, resulting in distribution into many different organs (mainly in the spleen and liver) rather than a large accumulation in kidneys. In addition, liposomal amphotericin B significantly changed the pharmacokinetic parameters, as reflected by higher $\mathrm{C}_{\max }$ and the area under the curve. ${ }^{62}$ Compared with conventional deoxycholate amphotericin B, the tolerated doses of amphotericin B-based nanoformulations improved from $1.5 \mathrm{mg} / \mathrm{kg}$ of body weight/day to $15 \mathrm{mg} /$ kg. ${ }^{63}$ These improvements reduced the toxicity without decreasing the therapeutic effect of amphotericin B, promoting the progress of drug delivery nanosystems for sepsis treatment. Detailed information of commercial nanoformulations for sepsis treatment is listed in Table 2.

\section{Limitations and Future Perspective}

Above all, it is still difficult to translate these new findings into clinical practice. The reasons were listed as below.

First, before entering the target site, the drug delivery nanosystem needs to overcome many obstacles, such as avoiding the reticuloendothelial system phagocytosis, ${ }^{86-88}$ and nanomaterial protein corona. ${ }^{89,90}$ 
Table 2 Commercial Nanoformulations for Sepsis Treatment

\begin{tabular}{|c|c|c|c|c|c|}
\hline Name & Company & Nanocomposition & Application & Clinical Stage & Ref. \\
\hline Abelcet & $\begin{array}{l}\text { Enzon Pharmaceutical } \\
\text { (Sigma-Tau } \\
\text { Pharmaceuticals) }\end{array}$ & Liposomal amphotericin B & Fungal infection & Commercially available & [64] \\
\hline AmBisome & Gilead Sciences & Liposomal amphotericin B & Fungal infection & Commercially available & {$[65,66]$} \\
\hline Amphotec & Sequus Pharmaceuticals & Liposomal amphotericin B & Fungal infection & Commercially available & [64] \\
\hline Fungisome & Lifecare Innovations & Liposomal amphotericin B & Fungal infection & Commercially available & {$[67$} \\
\hline Neulasta & Amgen Inc. & $\begin{array}{l}\text { Filgrastim-bound polymeric } \\
\text { NPs }\end{array}$ & $\begin{array}{l}\text { Fibrile } \\
\text { neutropenia }\end{array}$ & Commercially available & {$[68,69]$} \\
\hline LogiCath AgTive & $\begin{array}{l}\text { Smiths Medical } \\
\text { International }\end{array}$ & Nanosilver & $\begin{array}{l}\text { Antimicrobial } \\
\text { coating device }\end{array}$ & Commercially available & [70] \\
\hline PerOssal & Aap Impantate & $\begin{array}{l}\text { Calcium sulfate and } \\
\text { nanoparticulate } \\
\text { hydroxyapatite Composite }\end{array}$ & $\begin{array}{l}\text { Antibiotic } \\
\text { delivery }\end{array}$ & Commercially available & [7I] \\
\hline Spi-Argent & $\begin{array}{l}\text { Spire Biomedical } \\
\text { Corporation }\end{array}$ & Nanosilver & $\begin{array}{l}\text { Antimicrobial } \\
\text { coating device }\end{array}$ & Commercially available & [72-74] \\
\hline TAK-242 & $\begin{array}{l}\text { Takeda Global Research \& } \\
\text { Development Center, Inc. }\end{array}$ & Resatorvid emulsion & Sepsis & Commercially available & [75-77] \\
\hline PEV7 & Pevion Biotech Ltd & r-SAP2 virosomal vaccine & $\begin{array}{l}\text { Recurrent } \\
\text { vulvovaginal } \\
\text { candidiasis }\end{array}$ & Commercially available & {$[78,79]$} \\
\hline Cytosorb & $\begin{array}{l}\text { CytoSorbents } \\
\text { Corporation }\end{array}$ & Polymeric nanobeads & $\begin{array}{l}\text { Hemoadsorption } \\
\text { device for septic } \\
\text { shock }\end{array}$ & Commercially available & [80-84] \\
\hline MAT250I & Matinas Biopharm & $\begin{array}{l}\text { Amikacin-loaded lipid } \\
\text { nanocrystals }\end{array}$ & Bacterial infection & Commercially available & [85] \\
\hline
\end{tabular}

Note: Adapted from Papafilippou L, Claxton A, Dark P, Kostarelos K, Hadjidemetriou M. Nanotools for sepsis diagnosis and treatment. Adv Healthc Mater. 202I; I0(I): e200 I378. @ 2020 The Authors. Advanced Healthcare Materials published by Wiley-VCH GmbH ${ }^{57}$.

Second, overcoming bacterial resistance still complicate antibacterial drug delivery nanosystem preparation. For example, bacterial biofilm, a systematic bacterial growth organization, helps bacteria adapt to environmental pressure by adjusting their metabolism and developing strong drug resistance. ${ }^{91,92}$ How to use bacterial biofilms to infect a microenvironment with high acidity, hypoxia, and ROS content in tissues, and how to design a drug delivery nanosystem with high efficiency to destroy the biofilm still present some challenges. Additionally, although most bacteria are recognized and killed by phagocytes, some can survive in phagocytes. ${ }^{93}$ Therefore, how to construct phagocytes that can actively target the infected ones and realize antibiotics intracellular delivery should also be the focus of antibacterial drug delivery nanosystem development.

Third, the existing drug delivery nanosystem treatment modes are too unique. Sepsis is fierce, often starting from multiple pathways, destroying the body's function. ${ }^{94,95}$ Therefore, it is necessary to develop drug delivery nanosystems with more functions to kill bacteria, inhibit inflammatory cascades and monitor curative effects.

Overall, through continuous optimization, drug delivery nanosystems still present great potential for precise sepsis treatment.

\section{Abbreviations}

ICAM-1, Intercellular adhesion molecule 1; GO, Graphene oxide; ROS, Reactive oxygen species; RNS, Reactive nitrogen species; TMD, Transition Metal Dichalcogenide; CAT, Catalase; LPS, Lipopolysaccharide; TNF- $\alpha$, Tumor necrosis factor- $\alpha$; IL-1 $\beta$, IL-6, IL-10, Interleukin-1, interleukin-6, interleukin-10; HMGB1, High mobility group protein 1; iNOS, Inducible nitric oxide synthase; TCeria, Triphenylphosphine modified Cerium oxide nanoparticles; Atv/PTP-TCeria, Atorvastatin and TCeria were co-loaded into the core of ROS responsive grafts to construct the drug delivery nanosystems; miRNAs, Micro RNAs; PEI, Polyethylenimine; RNase A, Ribonuclease A; MIC, Minimal inhibit concentration; MDR, Multidrug resistant; 
PLGA, Poly lactic-co-glycolic acid; PA, Pulmonary ATRESIA; PT, Prothrombin time; WS2, Tungsten disulfide; MoSe2, Molybdenum Selenide; WSe2, Tungsten diselenide; BSA, bovine serum albumin; TLR-2, TLR-4, TLR-9, Toll-like receptor-2, Toll-like receptor-4, Toll-like receptor-9; NF kappa B, Nuclear factor kappa beta; Nrf2, Nuclear factor erythroid-2-related factor 2; SOD, Superoxide dismutase; cfDNA, Circulating free DNA; ICU, Intensive care unit; AmB, Amphotericin B deoxycholate; AmBisome, Amphotericin B; r-SAP2, Candida Albicans; Abelcet, Amphotericin B lipid comlex.

\section{Acknowledgments}

This work was supported in part by Construction of Chinese and Western medicine collaborative response and intervention platform for major critical medical events in Shanghai (ZY2018-2020-FWTX-7004) and Natural Science Foundation of Zhejiang Province (LQ21H300008).

\section{Disclosure}

The authors report no conflicts of interest in this work.

\section{References}

1. GBD 2017 Disease and Injury Incidence and Prevalence Collaborators. Global, regional, and national incidence, prevalence, and years lived with disability for 354 diseases and injuries for 195 countries and territories, 1990-2017: a systematic analysis for the Global Burden of Disease Study 2017. Lancet. 2018;392 (10159):1789-1858. doi:10.1016/S0140-6736(18)32279-7

2. Prescott HC, Angus DC. Enhancing recovery from sepsis: a review. JAMA. 2018;319(1):62-75. doi:10.1001/jama.2017.17687

3. Gauer R, Forbes D, Boyer N. Sepsis: diagnosis and management. Am Fam Physician. 2020;101(7):409-418.

4. Evans T. Diagnosis and management of sepsis. Clin Med. 2018;18 (2):146-149. doi:10.7861/clinmedicine.18-2-146

5. Rudd KE, Johnson SC, Agesa KM, et al. Global, regional, and national sepsis incidence and mortality, 1990-2017: analysis for the Global Burden of Disease Study. Lancet. 2020;395(10219):200-211. doi:10.1016/S0140-6736(19)32989-7

6. Thompson K, Venkatesh B, Finfer S. Sepsis and septic shock: current approaches to management. Intern Med J. 2019;49(2):160-170. doi:10.1111/imj.14199

7. Hunt A. Sepsis: an overview of the signs, symptoms, diagnosis, treatment and pathophysiology. Emerg Nurse. 2019;27:32-41. doi:10.7748/en.2019.e1926

8. Pant A, Mackraj I, Govender T. Advances in sepsis diagnosis and management: a paradigm shift towards nanotechnology. $J$ Biomed Sci. 2021;28(1):6. doi:10.1186/s12929-020-00702-6

9. Schrag SJ, Farley MM, Petit S, et al. Epidemiology of invasive early-onset neonatal sepsis, 2005 to 2014. Pediatrics. 2016;138(6): e20162013. doi:10.1542/peds.2016-2013

10. Popescu CR, Cavanagh MMM, Tembo B, et al. Neonatal sepsis in low-income countries: epidemiology, diagnosis and prevention. Expert Rev Anti Infect Ther. 2020;18(5):443-452. doi:10.1080/ 14787210.2020 .1732818
11. Chauhan N, Tiwari S, Jain U. Potential biomarkers for effective screening of neonatal sepsis infections: an overview. Microb Pathog. 2017;107:234-242. doi:10.1016/j.micpath.2017.03.042

12. Balayan S, Chauhan N, Chandra R, Kuchhal NK, Jain U. Recent advances in developing biosensing based platforms for neonatal sepsis. Biosens Bioelectron. 2020;169:112552. doi:10.1016/j.bios.2020.112552

13. Zahednezhad F, Saadat M, Valizadeh H, Zakeri-Milani P, Baradaran B. Liposome and immune system interplay: challenges and potentials. J Control Release. 2019;305:194-209. doi:10.1016/j. jconrel.2019.05.030

14. Patil YP, Jadhav S. Novel methods for liposome preparation. Chem Phys Lipids. 2014;177:8-18. doi:10.1016/j.chemphyslip.2013.10.011

15. De Jong WH, Borm PJ. Drug delivery and nanoparticles: applicationsand hazards. Int $J$ Nanomedicine. 2008;3(2):133-149. doi:10.2147/IJN.S596

16. Rajpoot K. Solid lipid nanoparticles: a promising nanomaterial in drug delivery. Curr Pharm Des. 2019;25(37):3943-3959. doi:10.2174/1381612825666190903155321

17. Lin M, Dai Y, Xia F, Zhang X. Advances in non-covalent crosslinked polymer micelles for biomedical applications. Mater Sci Eng C Mater Biol Appl. 2021;119:111626. doi:10.1016/j.msec.2020.111626

18. Jin X, Sun P, Tong G, Zhu X. Star polymer-based unimolecular micelles and their application in bio-imaging and diagnosis. Biomaterials. 2018;178:738-750. doi:10.1016/j.biomaterials.2 018.01 .051

19. Vader P, Mol EA, Pasterkamp G, Schiffelers RM. Extracellular vesicles for drug delivery. Adv Drug Deliv Rev. 2016;106(Pt A):148-156. doi:10.1016/j.addr.2016.02.006

20. Wang S, Gao J, Wang Z. Outer membrane vesicles for vaccination and targeted drug delivery. Wiley Interdiscip Rev Nanomed Nanobiotechnol. 2019;11(2):e1523. doi:10.1002/wnan.1523

21. Surman M, Drożdż A, Stępień E, Przybyło M. Extracellular vesicles as drug delivery systems - methods of production and potential therapeutic applications. Curr Pharm Des. 2019;25(2):132-154. doi: $10.2174 / 1381612825666190306153318$

22. Jin F, Liu D, Yu H, et al. Sialic acid-functionalized PEG-PLGA microspheres loading mitochondrial-targeting-modified curcumin for acute lung injury therapy. Mol Pharm. 2019;16(1):71-85. doi:10.1021/acs.molpharmaceut.8b00861

23. Dolman ME, Harmsen S, Storm G, Hennink WE, Kok RJ. Drug targeting to the kidney: advances in the active targeting of therapeutics to proximal tubular cells. Adv Drug Deliv Rev. 2010;62 (14):1344-1357. doi:10.1016/j.addr.2010.07.011

24. Liu D, Shu G, Jin F, et al. ROS-responsive chitosan-SS31 prodrug for AKI therapy via rapid distribution in the kidney and long-term retention in the renal tubule. Sci Adv. 2020;6(41):eabb7422. doi:10.1126/sciadv.abb7422

25. Fan X, Fan J, Wang X, Wu P, Wu G. S-thanatin functionalized liposome potentially targeting on Klebsiella pneumoniae and its application in sepsis mouse model. Front Pharmacol. 2015;6:249. doi:10.3389/fphar.2015.00249

26. Jiang S, Li S, Hu J, et al. Combined delivery of angiopoietin-1 gene and simvastatin mediated by anti-intercellular adhesion molecule-1 antibody-conjugated ternary nanoparticles for acute lung injury therapy. Nanomedicine. 2019;15(1):25-36. doi:10.1016/j.nano.201 8.08 .009

27. Qi J, Li W, Xu X, et al. Cyto-friendly polymerization at cell surfaces modulates cell fate by clustering cell-surface receptors. Chem Sci. 2020;11(16):4221-4225. doi:10.1039/C9SC06385D

28. Sadikot RT. The potential role of nano- and micro-technology in the management of critical illnesses. Adv Drug Deliv Rev. 2014;77:27-31. doi:10.1016/j.addr.2014.07.004

29. Kumar S, Tripathy S, Jyoti A, Singh SG. Recent advances in biosensors for diagnosis and detection of sepsis: a comprehensive review. Biosens Bioelectron. 2019;124-125:205-215. doi:10.1016/j. bios.2018.10.034 
30. Buxton DB. Nanomedicine for the management of lung and blood diseases. Nanomedicine. 2009;4(3):331-339. doi:10.2217/nnm.09.8

31. Zhang CY, Gao J, Wang Z. Bioresponsive nanoparticles targeted to infectious microenvironments for sepsis management. Adv Mater. 2018;30:e1803618. doi:10.1002/adma.201803618

32. Hou X, Zhang X, Zhao W, et al. Vitamin lipid nanoparticles enable adoptive macrophage transfer for the treatment of multidrug-resistant bacterial sepsis. Nat Nanotechnol. 2020;15(1):41-46. doi:10.1038/ s41565-019-0600-1

33. Ben-Akiva E, Meyer RA, Yu H, et al. Biomimetic anisotropic polymeric nanoparticles coated with red blood cell membranes for enhanced circulation and toxin removal. Sci Adv. 2020;6(16): eaay9035. doi:10.1126/sciadv.aay9035

34. Zheng H, Ma R, Gao M, et al. Antibacterial applications of graphene oxides: structure-activity relationships, molecular initiating events and biosafety. Sci Bull. 2018;63(2):133-142. doi:10.1016/j. scib.2017.12.012

35. Yim D, Lee DE, So Y, et al. Sustainable nanosheet antioxidants for sepsis therapy via scavenging intracellular reactive oxygen and nitrogen species. ACS Nano. 2020;14(8):10324-10336. doi:10.1021/ acsnano.0c03807

36. Weng Q, Sun H, Fang C, et al. Catalytic activity tunable ceria nanoparticles prevent chemotherapy-induced acute kidney injury without interference with chemotherapeutics. Nat Commun. 2021;12 (1):1436. doi:10.1038/s41467-021-21714-2

37. Kwon HJ, Cha MY, Kim D, et al. Mitochondria-targeting ceria nanoparticles as antioxidants for Alzheimer's disease. ACS Nano. 2016;10(2):2860-2870. doi:10.1021/acsnano.5b08045

38. Ni D, Wei H, Chen W, et al. Ceria nanoparticles meet hepatic ischemia-reperfusion injury: the perfect imperfection. Adv Mater. 2019;31(40):e1902956. doi:10.1002/adma.201902956

39. Kim J, Kim HY, Song SY, et al. Synergistic oxygen generation and reactive oxygen species scavenging by manganese ferrite/ceria co-decorated nanoparticles for rheumatoid arthritis treatment. ACS Nano. 2019;13(3):3206-3217. doi:10.1021/acsnano.8b08785

40. Chen G, Xu Y. Biosynthesis of cerium oxide nanoparticles and their effect on lipopolysaccharide (LPS) induced sepsis mortality and associated hepatic dysfunction in male Sprague Dawley rats. Mater Sci Eng C Mater Biol Appl. 2018;83:148-153. doi:10.1016/j. msec.2017.11.014

41. Yu H, Jin F, Liu D, et al. ROS-responsive nano-drug delivery system combining mitochondria-targeting ceria nanoparticles with atorvastatin for acute kidney injury. Theranostics. 2020;10(5):2342-2357. doi:10.7150/thno.40395

42. Zhang CY, Dong X, Gao J, Lin W, Liu Z, Wang Z. Nanoparticleinduced neutrophil apoptosis increases survival in sepsis and alleviates neurological damage in stroke. Sci Adv. 2019;5(11):eaax7964. doi:10.1126/sciadv.aax7964

43. Wang Z, Li J, Cho J, Malik AB. Prevention of vascular inflammation by nanoparticle targeting of adherent neutrophils. Nat Nanotechnol. 2014;9(3):204-210. doi:10.1038/nnano.2014.17

44. Wang J, Wang H, Zhu R, Liu Q, Fei J, Wang S. Anti-inflammatory activity of curcumin-loaded solid lipid nanoparticles in IL-1 $\beta$ transgenic mice subjected to the lipopolysaccharide-induced sepsis. Biomaterials. 2015;53:475-483. doi:10.1016/j.biomaterials.2015.02.116

45. Dawulieti J, Sun M, Zhao Y, et al. Treatment of severe sepsis with nanoparticulate cell-free DNA scavengers. Sci Adv. 2020;6(22): eaay7148. doi:10.1126/sciadv.aay7148

46. Mohr AM, Mott JL. Overview of microRNA biology. Semin Liver Dis. 2015;35(1):3-11. doi:10.1055/s-0034-1397344

47. Kemp JA, Kwon YJ. Cancer nanotechnology: current status and perspectives. Nano Converg. 2021;8(1):34. doi:10.1186/s40580-02100282-7

48. Revia RA, Stephen ZR, Zhang M. Theranostic nanoparticles for RNA-based cancer treatment. Acc Chem Res. 2019;52 (6):1496-1506. doi:10.1021/acs.accounts.9b00101
49. Jones Buie JN, Zhou Y, Goodwin AJ, et al. Application of deacetylated poly-N-acetyl glucosamine nanoparticles for the delivery of miR-126 for the treatment of cecal ligation and puncture-induced sepsis. Inflammation. 2019;42(1):170-184. doi:10.1007/s10753-0180882-8

50. Handa M, Sharma A, Verma RK, Shukla R. Polycaprolactone based nano-carrier for co-administration of moxifloxacin and rutin and its in-vitro evaluation for sepsis. J Drug Deliv Sci Technol. 2019;54:101286. doi:10.1016/j.jddst.2019.101286

51. Hassan A, Ikram A, Raza A, et al. Therapeutic potential of novel mastoparan-chitosan nanoconstructs against clinical MDR acinetobacter baumannii: in silico, in vitro and in vivo studies. Int J Nanomedicine. 2021;16:3755-3773. doi:10.2147/IJN.S296717

52. Galanzha EI, Shashkov E, Sarimollaoglu M, et al. In vivo magnetic enrichment, photoacoustic diagnosis, and photothermal purging of infected blood using multifunctional gold and magnetic nanoparticles. PLoS One. 2012;7(9):e45557. doi:10.1371/journal. pone.0045557

53. Kim JW, Galanzha EI, Zaharoff DA, Griffin RJ, Zharov VP. Nanotheranostics of circulating tumor cells, infections and other pathological features in vivo. Mol Pharm. 2013;10(3):813-830. doi: $10.1021 / \mathrm{mp} 300577 \mathrm{~s}$

54. Lykov AP. The therapeutic efficiency of mechanochemically synthesized compositions of nanostructured particles of silicium dioxide and antibiotics at experimental sepsis in $\left(\mathrm{CBA} \times \mathrm{C}_{57} \mathrm{Bl}_{6}\right) \mathrm{F} 1$ mice. Siberian Scientific Med J. 2012;32(3):21-26.

55. Shukla P, Verma AK, Dewangan J, et al. Chitosan coated curcumin nanocrystals augment pharmacotherapy via improved pharmacokinetics and interplay of $\mathrm{NF} \kappa \mathrm{B}$, Keap1 and Nrf2 expression in Gram negative sepsis. RSC $A d v$. 2015;5(70):57006-57020. doi:10.1039/ C5RA06786C

56. Liang Z, Yang Y, Yu G, et al. Engineering aluminum hydroxyphosphate nanoparticles with well-controlled surface property to enhance humoral immune responses as vaccine adjuvants. Biomaterials. 2021;275:120960. doi:10.1016/j.biomaterials.2021.120960

57. Papafilippou L, Claxton A, Dark P, Kostarelos K, Hadjidemetriou M. Nanotools for sepsis diagnosis and treatment. Adv Healthc Mater. 2021;10(1):e2001378. doi:10.1002/adhm.202001378

58. Farokhzad OC, Langer R. Impact of nanotechnology on drug delivery. ACS Nano. 2009;3(1):16-20. doi:10.1021/nn900002m

59. Bayda S, Adeel M, Tuccinardi T, Cordani M, Rizzolio F. The history of nanoscience and nanotechnology: from chemical-physical applications to nanomedicine. Molecules. 2019;25(1):112. doi:10.3390/ molecules25010112

60. Ellis D. Amphotericin B: spectrum and resistance. J Antimicrob Chemother. 2002;49(Suppl 1):7-10. doi:10.1093/jac/49.suppl_1.7

61. Alves D, Vaz AT, Grainha T, Rodrigues CF, Pereira MO. Design of an antifungal surface embedding liposomal amphotericin B through a mussel adhesive-inspired coating strategy. Front Chem. 2019;7:431. doi:10.3389/fchem.2019.00431

62. Groll AH, Rijnders BJA, Walsh TJ, Adler-Moore J, Lewis RE, Brüggemann RJM. Clinical pharmacokinetics, pharmacodynamics, safety and efficacy of liposomal amphotericin B. Clin Infect Dis. 2019;68(Suppl 4):S260-S274. doi:10.1093/cid/ciz076

63. Walsh TJ, Goodman JL, Pappas P, et al. Safety, tolerance, and pharmacokinetics of high-dose liposomal amphotericin B (AmBisome) in patients infected with Aspergillus species and other filamentous fungi: maximum tolerated dose study. Antimicrob Agents Chemother. 2001;45 (12):3487-3496. doi:10.1128/AAC.45.12.3487-3496.2001

64. Mocan L, Matea C, Tabaran FA, et al. Selective in vitro photothermal nano-therapy of MRSA infections mediated by IgG conjugated gold nanoparticles. Sci Rep. 2016;6:39466. doi:10.1038/srep39466

65. Stone NR, Bicanic T, Salim R, Hope W. Liposomal amphotericin B (AmBisome $\left.\left({ }^{\circledR}\right)\right)$ : a review of the pharmacokinetics, pharmacodynamics, clinical experience and future directions. Drugs. 2016;76 (4):485-500. doi:10.1007/s40265-016-0538-7 
66. Min Y, Caster JM, Eblan MJ, Wang AZ. Clinical translation of nanomedicine. Chem Rev. 2015;115(19):11147-11190. doi:10.1021/ acs.chemrev.5b00116

67. Zhu X, Radovic-Moreno AF, Wu J, Langer R, Shi J. Nanomedicine in the management of microbial infection-overview and perspectives. Nano Today. 2014;9(4):478-498. doi:10.1016/j.nantod.2014.06.003

68. Etheridge ML, Campbell SA, Erdman AG, Haynes CL, Wolf SM, McCullough J. The big picture on nanomedicine: the state of investigational and approved nanomedicine products. Nanomedicine. 2013;9(1):1-14. doi:10.1016/j.nano.2012.05.013

69. Farjadian F, Ghasemi A, Gohari O, Roointan A, Karimi M, Hamblin MR. Nanopharmaceuticals and nanomedicines currently on the market: challenges and opportunities. Nanomedicine. 2019;14 (1):93-126. doi: $10.2217 / \mathrm{nnm}-2018-0120$

70. Guggenbichler JP. Central venous catheter associated infections pathophysiology, incidence, clinical diagnosis, and prevention-a review. Materwiss Werksttech. 2003;34:1145-1154. doi:10.1002/ mawe. 200300712

71. Rauschmann MA, Wichelhaus TA, Stirnal V, et al. Nanocrystalline hydroxyapatite and calcium sulphate as biodegradable composite carrier material for local delivery of antibiotics in bone infections. Biomaterials. 2005;26(15):2677-2684. doi:10.1016/j.biomaterials.2004.06.045

72. Wijnhoven SWP, Peijnenburg W, Herberts CA, et al. Nano-silver-a review of available data and knowledge gaps in human and environmental risk assessment. Nanotoxicology. 2009;3(2):109-138. doi:10.1080/17435390902725914

73. Kampf G, Dietze B, Grosse-Siestrup C, Wendt C, Martiny H. Microbicidal activity of a new silver-containing polymer, SPI-ARGENT II. Antimicrob Agents Chemother. 1998;42 (9):2440-2442. doi:10.1128/AAC.42.9.2440

74. Massè A, Bruno A, Bosetti M, Biasibetti A, Cannas M, Gallinaro P. Prevention of pin track infection in external fixation with silver coated pins: clinical and microbiological results. J Biomed Mater Res. 2000;53(5):600-604. doi:10.1002/1097-4636(200009) 53:5<600::AID-JBM21>3.0.CO;2-D

75. Rice TW, Wheeler AP, Bernard GR, et al. A randomized, double-blind, placebo-controlled trial of TAK-242 for the treatment of severe sepsis. Crit Care Med. 2010;38(8):1685-1694. doi:10.1097/ CCM.0b013e3181e7c5c9

76. Bhattacharyya S, Wang W, Tamaki Z, et al. Pharmacological inhibition of toll-like receptor-4 signaling by TAK 242 prevents and induces regression of experimental organ fibrosis. Front Immunol. 2018;9:2434. doi:10.3389/fimmu.2018.02434

77. Sha T, Sunamoto M, Kitazaki T, Sato J, Ii M, Iizawa Y. Therapeutic effects of TAK-242, a novel selective Toll-like receptor 4 signal transduction inhibitor, in mouse endotoxin shock model. Eur J Pharmacol. 2007;571(2-3):231-239. doi:10.1016/j.ejphar.2007.06.027

78. Segal E. Testing antifungal vaccines in an animal model of invasive candidiasis and in human mucosal candidiasis. Methods Mol Biol. 2017; 1625:343-353.

79. Gov. Safety and immunogenicity study of a virosomal vaccine against recurrent vulvovaginal candida infection (clinical trial). Available from:https://clinicaltrials.gov/ct2/show/NCT01067131. NLM identifier: NCT01067131. Accessed February 11, 2010.

80. Hinz B, Jauch O, Noky T, Friesecke S, Abel P, Kaiser R. CytoSorb, a novel therapeutic approach for patients with septic shock: a case report. Int J Artif Organs. 2015;38(8):461-464. doi:10.5301/ ijao.5000429
81. Brouwer WP, Duran S, Kuijper M, Ince C. Hemoadsorption with CytoSorb shows a decreased observed versus expected 28-day all-cause mortality in ICU patients with septic shock: a propensity-score-weighted retrospective study. Crit Care. 2019;23 (1):317. doi:10.1186/s13054-019-2588-1

82. Mehta Y, Mehta C, Kumar A, et al. Experience with hemoadsorption $\left(\right.$ CytoSorb $\left.^{\circledR}\right)$ in the management of septic shock patients. World J Crit Care Med. 2020;9(1):1-12. doi:10.5492/wjccm.v9.i1.1

83. Schädler D, Pausch C, Heise D, et al. The effect of a novel extracorporeal cytokine hemoadsorption device on IL-6 elimination in septic patients: a randomized controlled trial. PLoS One. 2017;12 (10):e0187015. doi:10.1371/journal.pone.0187015

84. Friesecke S, Stecher SS, Gross S, Felix SB, Nierhaus A. Extracorporeal cytokine elimination as rescue therapy in refractory septic shock: a prospective single-center study. J Artif Organs. 2017;20(3):252-259. doi:10.1007/s10047-017-0967-4

85. Caster JM, Patel AN, Zhang T, Wang A. Investigational nanomedicines in 2016: a review of nanotherapeutics currently undergoing clinical trials. Wiley Interdiscip Rev Nanomed Nanobiotechnol. 2017;9(1):e1416. doi:10.1002/wnan.1416

86. Wang L, Chen S, Pei W, Huang B, Niu C. Magnetically targeted erythrocyte membrane coated nanosystem for synergistic photothermal/chemotherapy of cancer. J Mater Chem B. 2020;8 (18):4132-4142. doi:10.1039/D0TB00364F

87. Xia Q, Zhang Y, Li Z, Hou X, Feng N. Red blood cell membrane-camouflaged nanoparticles: a novel drug delivery system for antitumor application. Acta Pharm Sin B. 2019;9(4):675-689. doi:10.1016/j.apsb.2019.01.011

88. Tang Y, Wang X, Li J, et al. Overcoming the reticuloendothelial system barrier to drug delivery with a "Don't-Eat-Us" strategy. ACS Nano. 2019;13(11):13015-13026. doi:10.1021/acsnano.9b05679

89. Lu X, Xu P, Ding HM, Yu YS, Huo D, Ma YQ. Tailoring the component of protein Corona via simple chemistry. Nat Commun. 2019;10(1):4520. doi:10.1038/s41467-019-12470-5

90. Elechalawar CK, Hossen MN, McNally L, Bhattacharya R, Mukherjee P. Analysing the nanoparticle-protein Corona for potential molecular target identification. J Control Release. 2020;322:122-136. doi:10.1016/j.jconrel.2020.03.008

91. Yan J, Bassler BL. Surviving as a community: antibiotic tolerance and persistence in bacterial biofilms. Cell Host Microbe. 2019;26 (1):15-21. doi:10.1016/j.chom.2019.06.002

92. Grande R, Puca V, Muraro R. Antibiotic resistance and bacterial biofilm. Expert Opin Ther Pat. 2020;30(12):897-900. doi:10.1080/ 13543776.2020.1830060

93. Yang S, Tang Q, Chen L, et al. Cationic lipid-based intracellular delivery of bacterial effectors for rewiring malignant cell signaling. Angew Chem Int Ed Engl. 2020;59(41):18087-18094. doi:10.1002/ anie. 202009572

94. Uhle F, Lichtenstern C, Brenner T, Weigand MA. Pathophysiology of sepsis. Anasthesiol Intensivmed Notfallmed Schmerzther. 2015;50 (2):114-122. doi:10.1055/s-0041-100391

95. Salomão R, Ferreira BL, Salomão MC, Santos SS, Azevedo LCP, Brunialti MKC. Sepsis: evolving concepts and challenges. Braz J Med Biol Res. 2019;52(4):e8595. doi:10.1590/1414-431×20198595 


\section{Publish your work in this journal}

The Journal of Inflammation Research is an international, peerreviewed open-access journal that welcomes laboratory and clinica findings on the molecular basis, cell biology and pharmacology of inflammation including original research, reviews, symposium reports, hypothesis formation and commentaries on: acute/chronic inflammation; mediators of inflammation; cellular processes; molecular mechanisms; pharmacology and novel anti-inflammatory drugs; clinical conditions involving inflammation. The manuscript management system is completely online and includes a very quick and fair peerreview system. Visit http://www.dovepress.com/testimonials.php to read real quotes from published authors.

Submit your manuscript here: https://www.dovepress.com/journal-of-inflammation-research-journal 